\title{
Small-Molecule Biomarkers for Clinical PET Imaging of Apoptosis
}

\author{
Ayelet Reshef ${ }^{1}$, Anat Shirvan ${ }^{1}$, Ayelet Akselrod-Ballin², Anders Wall ${ }^{3}$, and Ilan Ziv ${ }^{1}$ \\ ${ }^{\text {I} A p o s e n s e ~ L t d ., ~ P e t a c h ~ T i k v a, ~ I s r a e l ; ~}{ }^{2}$ Computational Radiology, Children's Hospital, Harvard Medical School, Boston, Massachusetts; \\ and ${ }^{3}$ Imanet, Uppsala, Sweden
}

\begin{abstract}
Apoptosis is a fundamental biologic process. Molecular imaging of apoptosis in vivo may have important implications for clinical practice, assisting in early detection of disease, monitoring of disease course, assessment of treatment efficacy, or development of new therapies. Although a PET probe for clinical imaging of apoptosis would be highly desirable, this is yet an unachieved goal, mainly because of the required challenging integration of various features, including sensitive and selective detection of the apoptotic cells, clinical aspects such as favorable biodistribution and safety profiles, and compatibility with the radiochemistry and imaging routines of clinical PET centers. Several approaches are being developed to address this challenge, all based on novel small-molecule structures targeting various steps of the apoptotic cascade. This novel concept of smallmolecule PET probes for apoptosis is the focus of this review.
\end{abstract}

Key Words: PET; molecular imaging; apoptosis; small-molecule

J Nucl Med 2010; 51:837-840

DOI: 10.2967/jnumed.109.063917

The discovery of apoptosis is one of the most important advances in biologic research of recent years. The concept that cell death is not merely random and chaotic but rather is programmed and regulated sparked imagination and revolutionized biologic thinking. Intensive research unveiled apoptosis as a universal and fundamental process inherent in any nucleated cell and tightly regulated and executed by specific, genetically controlled cellular protein systems. Numerous triggers, either physiologic or disease-related, can trigger the apoptotic program, all ultimately converging into a point at which the cell commits to the death process. Apoptotic cells are converted into clusters of membrane-bound particles that are subsequently engulfed and eliminated by macrophages $(1,2)$. Two major pathways of apoptosis have been characterized: the intrinsic pathway, featuring mitochondrial permeability transition, irreversible membrane depolarization, release of cytochrome $\mathrm{c}$, and activation of caspases, and the extrinsic pathway, featuring transmembrane "death receptors" with respective ligands (e.g., tumor necrosis factor, tumor

Received Sep. 18, 2009; revision accepted Jan. 5, 2010.

For correspondence or reprints contact: Ilan Ziv, Aposense Ltd., 5 Odem

St., P.O. Box 7119, Petach Tikva, 49170, Israel.

E-mail: ilan@aposense.com

COPYRIGHT @ 2010 by the Society of Nuclear Medicine, Inc. necrosis factor-related apoptosis-inducing ligand, and Fas ligand) and signal transduction pathways, leading to caspase activation (1).

\section{CLINICAL PERSPECTIVE ON APOPTOSIS IMAGING}

From a clinical viewpoint, apoptosis research creates exciting opportunities. A biomarker for apoptosis may assist in early detection of disease, monitoring of disease course, or assessment of the effect of therapy on a wide scope of diseases. However, to date, the goal of imaging the death process in vivo as part of routine clinical practice has not been achieved. A useful probe for clinical molecular imaging of apoptosis should successfully integrate several distinct features (Table 1). First, the probe should manifest high selectivity and specificity for apoptotic cells, detect cells in the early stages of the death process, and preferably distinguish between apoptotic and necrotic cells. Second, the probe should be suitable for use in vivo. Primarily, this implies adequate biodistribution on intravenous administration, rapid distribution throughout the body, detection of the apoptotic cells in vivo, and rapid clearance from nontarget sites, with a favorable signal-to-background ratio. Third, the probe should be nontoxic and chemically and immunologically inert. Fourth, the probe should have high stability in vivo, with minimal metabolism (3). Finally, the probe should be compatible with routine clinical practice. PET, because of its advantages in image quality and signal quantification, emerges as the leading modality in molecular imaging but presents challenges regarding rapid and efficient radiolabeling. Taken together, these considerations demonstrate the complexity of translating into reality the vision of molecular imaging of apoptosis by PET.

\section{PROTOTYPE BIOMARKER FOR APOPTOSIS}

The first biomarker for apoptosis evaluated clinically was ${ }^{99 \mathrm{~m}} \mathrm{Tc}$-annexin-V. This $36-\mathrm{kDa}$ protein binds with high affinity to phosphatidylserine head groups, thereby reporting on phosphatidylserine exposure on the cell surface, an early and universal event in apoptosis. Although initially used to detect apoptosis in vitro, ${ }^{99 \mathrm{~m}}$ Tc-labeled derivatives of annexin- $\mathrm{V}$ allowed extension to in vivo indications. Pioneering SPECT studies with ${ }^{99 \mathrm{~m}} \mathrm{Tc}$-labeled annexin-V provided a highly important proof of concept for the feasibility and potential clinical utility of apoptosis imaging in various medical disorders (4). ${ }^{99 m} \mathrm{Tc}$-annexin-V was found useful for detecting apoptosis in cardiovascular disorders such as myocardial infarction or atherosclerotic plaques and for monitoring the effect of therapy 
TABLE 1. Requirements of a PET Probe for Imaging

Apoptosis

Requirement

Detection of apoptotic cells

\begin{tabular}{|c|c|}
\hline $\begin{array}{l}\text { Adequate performance } \\
\text { in vivo }\end{array}$ & $\begin{array}{l}\text { High stability on administration } \\
\text { in vivo } \\
\text { Rapid distribution on } \\
\text { administration, with rapid } \\
\text { clearance from nontarget sites, } \\
\text { preferably through urine } \\
\text { Adequate dosimetry } \\
\text { Safety: lack of chemical or } \\
\text { immunologic reactivity }\end{array}$ \\
\hline $\begin{array}{l}\text { Compatibility with } \\
\text { clinical practice }\end{array}$ & $\begin{array}{l}\text { Rapid, robust, and reproducible } \\
\text { radiolabeling procedure; } \\
\text { preferably with }{ }^{18} \mathrm{~F} \\
\text { Good integration in the routine } \\
\text { radiochemistry and imaging } \\
\text { procedures of clinical PET } \\
\text { centers }\end{array}$ \\
\hline
\end{tabular}

in certain types of cancer, such as tumors of the head and neck $(5,6)$. However, these studies also delineated limitations of such protein-based molecular imaging (1). At the level of specificity, annexin-V labels both apoptotic and necrotic cells by also reaching and binding to intracellular phosphatidylserine through disrupted plasma membranes. At the level of biodistribution, annexin- $\mathrm{V}$ is characterized by relatively slow clearance from nontargeted tissues, conceivably because of its large protein structure, thus reducing signal-to-noise ratios (1).

Radiolabeling of annexin-V for PET imaging was also attempted. The 110 -min half-life of ${ }^{18} \mathrm{~F}$, the most widely used isotope for PET, dictates a need for rapid and efficient radiolabeling. Because annexin- $\mathrm{V}$ harbors numerous functional groups requiring protection and deprotection steps, its direct radiolabeling with ${ }^{18} \mathrm{~F}$ is impractical. Labeling groups, such as $\mathrm{N}$-succinimidyl $4-{ }^{18} \mathrm{~F}$-fluorobenzoate, have therefore been synthesized, with subsequent derivatization of annexin-V (7). Albeit obtaining good synthetic yields, radiolabeling and purification of such probes are quite complex, especially in the context of routine clinical practice. To allow longer radiolabeling procedures and also address the slow clearance rate of annexin- $\mathrm{V}$, alternative radioisotopes with a longer halflife than that of ${ }^{18} \mathrm{~F}$ (e.g., ${ }^{64} \mathrm{Cu}$ or ${ }^{124} \mathrm{I}$, with half-lives of $12.7 \mathrm{~h}$ and $4.2 \mathrm{~d}$, respectively) were also investigated $(2,8)$. However, these isotopes are not routinely used in clinical PET.

\section{SMALL-MOLECULE PET PROBES FOR APOPTOSIS}

Conceptually, low-molecular-weight compounds may have advantages as PET tracers over large protein-based probes $(2,4)$. One major potential advantage of small-molecule compounds is that radiolabeling procedures are more feasible. This greater feasibility may relate, in part, to the small number of functional groups in such compounds, compared with large proteins. Thus, avenues are opened for direct, rapid, and robust attachment of the radioisotope, a key requirement for clinical practice. Other important features of small-molecule PET probes include potentially better biodistribution and clearance profiles. Small molecules are also less prone to elicit immunologic responses. Importantly, small molecules are also more suitable for structural modifications, which are often necessary to optimize probe performance. The path to the desirable small-molecule PET probes for apoptosis will conceivably extend beyond direct phosphatidylserine ligands such as annexin- $\mathrm{V}$ and will probably have to rely also on other mechanisms of action. The small moiety of the phosphatidylserine head group cannot function by itself as a pharmacophore to which a small-molecule probe can bind effectively and selectively, as reflected, among others, by the fact that each annexin- $\mathrm{V}$ molecule binds to numerous phosphatidylserine head groups in order to generate enough free energy for binding (1). Several approaches have been reported, based on targeting other steps or hallmarks of the apoptotic cascade rather than direct phosphatidylserine binding. These reports represent the current 3 major approaches to addressing the challenge of small-molecule PET probes for apoptosis: probes for caspase activation; probes for detection of collapse of mitochondrial membrane potential; and detection of the apoptotic membrane imprint, that is, a complex of apoptosis-specific membrane alterations distinguishing the apoptotic cell from its viable or necrotic counterparts.

\section{PET PROBES FOR CASPASE ACTIVATION}

Caspases play pivotal roles in both the intrinsic and the extrinsic pathways of apoptosis. Several groups have investigated potential ligands for activated capase-3. Although research initially focused on peptide-based ligands, a major advance has recently been made with the introduction of novel, small-molecule caspase inhibitors, based mostly on the isatin sulfonamide moiety. Several compounds of this class have been radiolabeled with either ${ }^{11} \mathrm{C}$ or ${ }^{18} \mathrm{~F}$ for PET. These include the isatin compounds ${ }^{18} \mathrm{~F}$-ICMT $11,{ }^{18} \mathrm{~F}$-caspase binding radioligand, ${ }^{11} \mathrm{C}-\mathrm{WC}-98$, and ${ }^{18} \mathrm{~F}-\mathrm{WC}-\mathrm{IV}-3$, which were examined as apoptosis probes either in cell cultures or in vivo (9-14). All demonstrated high affinity to caspase- 3 in isolated enzymatic assays and have shown increased uptake in models of apoptosis, such as liver cell apoptosis induced by either cycloheximide or anti-Fas antibody, with confirmatory histologic assessment of the death process. A concern that emerged about these compounds is the apparent substantial drop in probe affinity on moving from the isolated protein system to whole-cell or in vivo settings, where markedly higher concentrations of the isatin compounds are needed to inhibit caspase $(14,15)$. This observation, and an apparently suboptimal biodistribution profile, may relate in part to a potential nonspecific chemical reactivity of the dicarbonyl moiety of the isatin molecule. Although this functionality is essential for probe binding to the nucleophilic cysteine thiolate at the caspase-active site, it may also lead to nonspecific binding to other cysteine proteases (e.g., cathepsins) that manifest constitutive expression or to various other macromolecules in the body that contain nucleophilic moieties such as amine or thiol groups (15). Considering the universal role of 
caspases in apoptosis, and especially that of capsase- 3 in the final common pathway of the death program, targeting activated caspases is clearly an attractive approach that may be expected to develop into clinical trials, pending further optimization of probe structure and performance.

\section{PET PROBES FOR DETECTION OF COLLAPSE OF MITOCHONDRIAL MEMBRANE POTENTIAL}

The permanent collapse of the mitochondrial membrane electrochemical potential plays a key role in apoptosis. A recently explored approach uses radiolabeled voltage-sensitive probes, that is, ${ }^{18} \mathrm{~F}$-radiolabeled organic cations such as ${ }^{18} \mathrm{~F}$-fluorobenzyl triphenylphosphonium cation $\left({ }^{18} \mathrm{~F}-\mathrm{FBnTP}\right)$, for apoptosis imaging via detection of the loss of mitochondrial membrane potential. The electrochemical proton gradient across the inner mitochondrial membrane in viable cells favors inward movement of such cations into the mitochondrial matrix. This gradient is lost early on induction of apoptosis, with a respective reduction in tracer uptake. Therefore, these probes manifest signal reduction on induction of the death process, in contrast to the increased signal observed with the other probe categories. Significant respective reductions in ${ }^{18} \mathrm{~F}$-FBnTP uptake on induction of apoptosis have been demonstrated in vitro in staurosporine-treated lung carcinoma cells (16) and paclitaxel-treated breast carcinoma cells (17) and in vivo in prostate tumor-bearing mice treated with docetaxel (17). A potential limitation of this approach, which merits further research, is a potential efflux of these probes from certain living cells by multidrug-resistance proteins. Viable cells manifesting such a probe efflux may be misdiagnosed as apoptotic cells $(16,17)$.

\section{DETECTION OF APOPTOTIC MEMBRANE IMPRINT}

The apoptotic membrane imprint is a complex of cellular alterations that take place early in the apoptotic process. The imprint comprises an irreversible loss of plasma membrane potential, permanent acidification of the external plasma membrane leaflet and cytosol, and activation of the membrane phospholipid scramblase system, with preservation of membrane integrity (Fig. 1D). The concerted concurrence of this complex of cellular features distinguishes the apoptotic cell from its viable or necrotic counterparts (18). A set of novel small-molecule probes designated the Aposense compounds (a patented platform technology; Aposense Ltd.) has been rationally designed to detect this unique, apoptosis-related complex of cellular alterations, with consequent selective accumulation within apoptotic cells driven by the apoptotic scramblase activation, irreversible membrane depolarization, and cellular acidification (18). Accordingly, the performance of several Aposense compounds (e.g., DDC, ML-10, ML-9, NST-732, and NST-729) has been reported. These compounds have shown activity in various preclinical models of disease such as apoptosis induced by anticancer agents in tumors (19-21), models of renal failure (22), and neurovascular apoptosis in ischemic cerebral stroke (23) and neurodegenerative diseases (24). Independently, Zeng et al. described the development of an ${ }^{18} \mathrm{~F}$-labeled dansylhydrazone derivative of the Aposense compound NST-732 capable of detecting chemotherapy-induced apoptosis in tissue culture (25). The PET tracer ${ }^{18}$ F-ML-10 (Fig. 1A) is a highly compact compound (molecular weight, 206) in the Aposense family. It shows selective uptake by apoptotic cells, in correlation with the apoptotic hallmarks of breakdown of mitochondrial membrane potential, caspase activation, or apoptotic DNA fragmentation. Signal is lost on membrane rupture, and thus ${ }^{18} \mathrm{~F}-\mathrm{ML}-10$ is capable of distinguishing between apoptotic and necrotic cells (18). ${ }^{18} \mathrm{~F}-\mathrm{ML}-10$ is the first PET tracer for apoptosis that has been advanced into the clinical stage of development, with promising results to date in several small-scale clinical trials. In a phase I trial on healthy volunteers, ${ }^{18}$ F-ML-10 manifested high stability in vivo and favorable

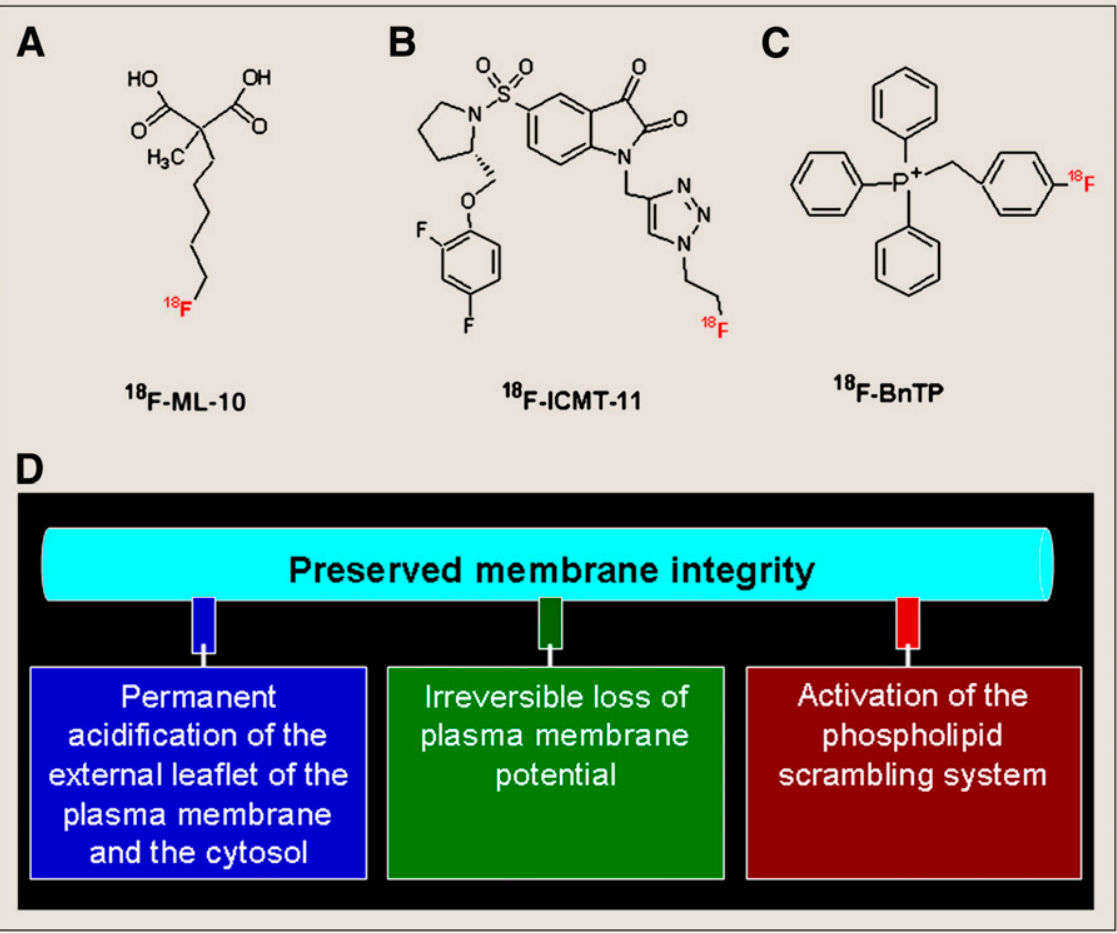

FIGURE 1. (A-C) ${ }^{18}$ F-labeled, smallmolecule PET probes for apoptosis: ${ }^{18} \mathrm{~F}-\mathrm{ML}-10$ (A), ${ }^{18} \mathrm{~F}-\mathrm{ICMT}-11 \quad(\mathrm{~B})$, and ${ }^{18} \mathrm{~F}-\mathrm{FBnTP}$ (C). (D) Scheme of apoptotic membrane imprint: complex of cellular alterations, occurring early on induction of apoptosis, that distinguish apoptotic cell from its viable or necrotic counterparts. 


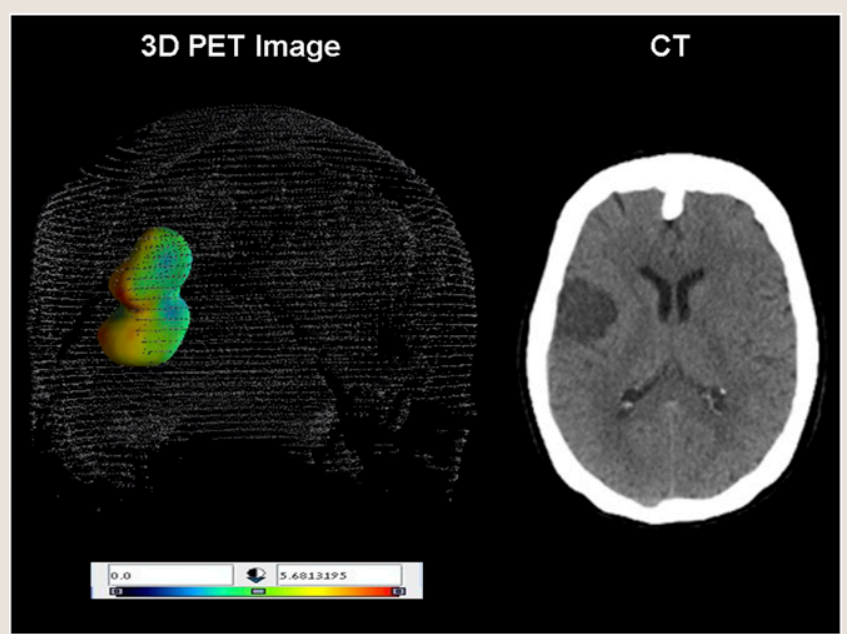

FIGURE 2. PET image with ${ }^{18} \mathrm{~F}-\mathrm{ML}-10$ in acute ischemic cerebral stroke. Tridimensional presentation of summed PET image obtained after administration of ${ }^{18} \mathrm{~F}-\mathrm{ML}-10$ to patient with acute ischemic cerebral stroke (day 3 after onset). Various intensities of apoptotic cell death are seen within region of infarct. Respective CT scan is on right.

biodistribution and dosimetry profiles. In a phase IIa study, PET imaging of apoptosis of neurovascular cells has been achieved in patients with acute ischemic cerebral stroke, as exemplified in Figure 2. In patients with brain metastases treated with whole-brain radiation therapy, PET imaging with ${ }^{18} \mathrm{~F}-\mathrm{ML}-10$ provided early detection of tumor response to treatment, which was predictive of an anatomic response evident on MRI two months later (A.M. Allen, unpublished data, December 2009). Further expansion of the clinical experience with this novel probe is under way.

\section{TRENDS AND FUTURE PROSPECTS}

Clinical imaging of apoptosis has undergone a dynamic evolution over the last decade. Following proof-of-concept studies

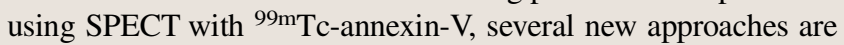
being developed to enable PET imaging of the death process. These innovative approaches extend beyond the initial concept of phosphatidylserine-directed, protein-based probes represented by annexin- $\mathrm{V}$ and are based on novel small-molecule structures detecting new targets along the apoptotic cascade. Such strategies may address the complex set of features required to qualify a probe for imaging apoptosis in clinical practice. Importantly, these development efforts are concurrent with impressive advances in the fields of imaging equipment, radiolabeling techniques for PET, and mathematic algorithms for quantitative analysis of the PET signal. We believe that the integration of all these disciplines may be the key to successful introduction of noninvasive imaging of apoptosis into routine clinical practice, an important step toward its anticipated future role in patient care.

\section{ACKNOWLEDGMENT}

Drs. Reshef, Shirvan, and Ziv are employees of Aposense Ltd., which holds the patent for the Aposense platform technology.

\section{REFERENCES}

1. Blankenberg FG. In vivo detection of apoptosis. J Nucl Med. 2008;49(suppl 2):81S-95S.

2. De Saint-Hubert M, Prinsen K, Mortelmans L, Verbruggen A, Mottaghy FM. Molecular imaging of cell death. Methods. 2009;48:178-187.

3. Jaffer FA, Weissleder R. Molecular imaging in the clinical arena. JAMA. 2005;293:855-862.

4. Tait JF. Imaging of apoptosis. J Nucl Med. 2008;49:1573-1576.

5. Wolters SL, Corsten MF, Reutelingsperger CP, Narula J, Hofstra L. Cardiovascular molecular imaging of apoptosis. Eur J Nucl Med Mol Imaging. 2007;34(suppl 1):S86-S98.

6. Hoebers FJ, Kartachova M, de Bois J, et al. ${ }^{99 \mathrm{~m}} \mathrm{Tc}$ Hynic-rh-annexin V scintigraphy for in vivo imaging of apoptosis in patients with head and neck cancer treated with chemoradiotherapy. Eur J Nucl Med Mol Imaging. 2008;35:509-518.

7. Yagle KJ, Eary JF, Tait JF, et al. Evaluation of ${ }^{18} \mathrm{~F}$-annexin V as a PET imaging agent in an animal model of apoptosis. J Nucl Med. 2005;46:658-666.

8. Cauchon N, Langlois R, Rousseau JA, et al. PET imaging of apoptosis with ${ }^{64} \mathrm{Cu}$-labeled streptavidin following pretargeting of phosphatidylserine with biotinylated annexin-V. Eur J Nucl Med Mol Imaging. 2007;34:247-258.

9. Chen DL, Zhou D, Chu W, et al. Comparison of radiolabeled isatin analogs for imaging apoptosis with positron emission tomography. Nucl Med Biol. 2009; 36:651-658.

10. Nguyen QD, Smith G, Glaser M, Perumal M, Arstad E, Aboagye EO. Positron emission tomography imaging of drug-induced tumor apoptosis with a caspase3/7 specific [ ${ }^{18}$ F]-labeled isatin sulfonamide. Proc Natl Acad Sci USA. 2009; 106:16375-16380.

11. Podichetty AK, Wagner S, Schröer S, et al. Fluorinated isatin derivatives. Part 2. New N-substituted 5pyrrolidinylsulfonyl isatins as potential tools for molecular imaging of caspases in apoptosis. J Med Chem. 2009;52:3484-3495.

12. Zhou D, Chu W, Chen DL, et al. $\left[{ }^{18} \mathrm{~F}\right]-$ and $\left[{ }^{11} \mathrm{C}\right]$-labeled N-benzyl-isatin sulfonamide analogues as PET tracers for apoptosis: synthesis, radiolabeling mechanism, and in vivo imaging study of apoptosis in Fas-treated mice using [ ${ }^{11}$ C]WC-98. Org Biomol Chem. 2009; 7:1337-1348.

13. Zhou D, Chu W, Rothfuss J, et al. Synthesis, radiolabeling, and in vivo evaluation of an ${ }^{18} \mathrm{~F}$-labeled isatin analog for imaging caspase-3 activation in apoptosis. Bioorg Med Chem Lett. 2006;16:5041-5046.

14. Kopka K, Faust A, Keul P, et al. 5-pyrrolidinylsulfonyl isatins as a potential tool for the molecular imaging of caspases in apoptosis. J Med Chem. 2006;49:6704 6715 .

15. Lee D, Long SA, Murray JH, et al. Potent and selective nonpeptide inhibitors of caspases 3 and 7. J Med Chem. 2001;44:2015-2026.

16. Madar I, Ravert H, Nelkin B, et al. Characterization of membrane potentialdependent uptake of the novel PET tracer ${ }^{18} \mathrm{~F}$-fluorobenzyl triphenylphosphonium cation. Eur J Nucl Med Mol Imaging. 2007;34:2057-2065.

17. Madar I, Huang Y, Ravert H, et al. Detection and quantification of the evolution dynamics of apoptosis using the PET voltage sensor ${ }^{18} \mathrm{~F}$-fluorobenzyl triphenyl phosphonium. J Nucl Med. 2009;50:774-780.

18. Cohen A, Shirvan A, Levin G, Grimberg H, Reshef A, Ziv I. From the Gla domain to a novel small-molecule detector of apoptosis. Cell Res. 2009;19:625-637.

19. Cohen A, Ziv I, Aloya T, et al. Monitoring of chemotherapy-induced cell death in melanoma tumors by N,N'-didansyl-L-cystine. Technol Cancer Res Treat. 2007;6:221-234.

20. Aloya R, Shirvan A, Grimberg H, et al. Molecular imaging of cell death in vivo by a novel small molecule probe. Apoptosis. 2006;11:2089-2101.

21. Grimberg H, Levin G, Shirvan A, et al. Monitoring of tumor response to chemotherapy in vivo by a novel small-molecule detector of apoptosis. Apoptosis. 2009;14:257-267.

22. Damianovich M, Ziv I, Heyman SN, et al. ApoSense: a novel technology for functional molecular imaging of cell death in models of acute renal tubular necrosis. Eur J Nucl Med Mol Imaging. 2006;33:281-291.

23. Reshef A, Shirvan A, Waterhouse RN, et al. Molecular imaging of neurovascular cell death in experimental cerebral stroke by PET. J Nucl Med. 2008;49:15201528 .

24. Shirvan A, Reshef A, Yogev-Falach M, Ziv I. Molecular imaging of neurodegeneration by a novel cross-disease biomarker. Exp Neurol. 2009;219:274283.

25. Zeng W, Yao ML, Townsend D, et al. Synthesis, biological evaluation and radiochemical labeling of a dansylhydrazone derivative as a potential imaging agent for apoptosis. Bioorg Med Chem Lett. 2008;18:3573-3577. 